\title{
Tabela de vida de fertilidade de três espécies neotropicais de Trichogrammatidae em ovos de hospedeiros alternativos como critério de seleção hospedeira
}

\author{
Nivia da Silva Dias ${ }^{1}$, José Roberto Postali Parra ${ }^{1} \&$ Carlos Tadeu dos Santos Dias²
}

${ }^{1}$ Laboratório de Biologia de Insetos, Departamento de Entomologia e Acarologia, Esalq/USP, Av. Pádua Dias, 11, Caixa Postal 9, 13418-900 Piracicaba-SP, Brasil.dias.nivia@gmail.com; jrpparra@esalq.usp.br

${ }^{2}$ Departamento de Ciências Exatas, Esalq/USP. Av. Pádua Dias, 11, Caixa Postal 9, 13418-900 Piracicaba-SP, Brasil. ctsdias@esalq.usp.br

\begin{abstract}
Fertility life table of three neotropical species of Trichogrammatidae on factitious hosts as a criterion for selection of hosts. The objective of this work was to select the factitious host that permit the best development of three neotropical Trichogrammatidae species, Trichogramma atopovirilia Oatman \& Platner, 1983; Trichogramma bruni Nagaraja, 1983, and Trichogrammatoidea annulata De Santis, 1972, using the fertility life table on their respective hosts as a comparative parameter. Mean generation time $(T)$, net reproductive rate $\left(R_{0}\right)$, intrinsic rate of natural increase $\left(r_{m}\right)$ and the finite rate of increase $(\lambda)$ were estimated. A fertility life table is useful to select the most adequate factitious hosts for the trichogrammatid species. Corcyra cephalonica (Stainton, 1865) (Lepidoptera, Pyralidae) was the most suitable factitious host for rearing of T. annulata and T. bruni; while Anagasta kuehniella (Zeller, 1879) (Lepidoptera, Pyralidae) and/or C. cephalonica were the most suitable hosts for $T$. atopovirilia. Sitotroga cerealella (Olivier, 1819) (Lepidoptera, Gelechiidae) presented a low capacity of population increase for the three species of parasitoids, therefore, being an inadequate species as a factitious host.
\end{abstract}

KEYWORDS. Egg parasitoid; factitious host; life table; Trichogramma spp.

RESUMO. Tabela de vida de fertilidade de três espécies neotropicais de Trichogrammatidae em ovos de hospedeiros alternativos como critério de seleção hospedeira. O objetivo deste trabalho foi selecionar o hospedeiro alternativo que permita o melhor desenvolvimento das três espécies neotropicais de tricogramatídeos, Trichogramma atopovirilia Oatman \& Platner, 1983; Trichogramma bruni Nagaraja, 1983 e Trichogrammatoidea annulata De Santis, 1972, utilizando-se como parâmetro comparativo as tabelas de vida de fertilidade nos respectivos hospedeiros. Foram estimados a duração média de uma geração (T), taxa líquida de reprodução $\left(\mathrm{R}_{\mathrm{o}}\right)$, razão infinitesimal $\left(\mathrm{r}_{\mathrm{m}}\right)$ e a razão finita de aumento $(\lambda)$. A tabela de vida de fertilidade pode ser utilizada para selecionar o hospedeiro alternativo mais adequado para as espécies de tricogramatídeos. Corcyra cephalonica (Stainton, 1865) (Lepidoptera, Pyralidae) foi o hospedeiro alternativo mais adequado para criação de T. annulata e de T. bruni, enquanto que para T. atopovirilia, Anagasta kuehniella (Zeller, 1879) (Lepidoptera, Pyralidae) e/ou C. cephalonica foram os hospedeiros mais adequados. Sitotroga cerealella (Olivier, 1819) (Lepidoptera, Gelechiidae) apresentou baixa capacidade de aumento populacional para as três espécies de parasitóides, sendo, portanto, uma espécie inadequada como hospedeiro alternativo para as mesmas.

PALAVRAS-CHAVE. Hospedeiro alternativo; parasitóides de ovos; tabela de vida; Trichogramma spp.

A grande importância dos parasitóides do gênero Trichogramma em programas de Controle Biológico (Parra 1997) justifica a busca incessante de técnicas de criação eficientes para sua produção em larga escala.

Estes parasitóides têm sido produzidos em hospedeiros alternativos, para reduzir os custos e aumentar a eficiência durante o processo de produção massal. No entanto, até mesmo para um parasitóide polífago, a adequabilidade destes hospedeiros alternativos pode ser variável. Características como volume do ovo hospedeiro, espessura do córion, conteúdo nutricional, idade e forma de postura dos hospedeiros podem afetar a qualidade dos parasitóides, bem como a porcentagem de parasitismo, razão sexual e o número de parasitóides/ ovo do hospedeiro (Hoffmann et al. 2001; Roriz et al. 2006; Rukmowati-Brotodjojo \& Walter 2006). Desta forma, o desempenho do parasitóide está relacionado à qualidade do hospedeiro (Bai et al. 1992; Schmidt 1994), sendo a escolha do hospedeiro de criação de suma importância na etapa de criação em laboratório, pois a escolha inadequada poderá comprometer o programa de controle biológico.

Trichogramma spp. têm sido criados massalmente em ovos de Sitotroga cerealella (Olivier, 1819) (Lepidoptera, Gelechiidae), Anagasta kuehniella (Zeller, 1879) (Lepidoptera, Pyralidae) e Corcyra cephalonica (Stainton, 1865) Lepidoptera, Pyralidae) devido à disponibilidade e facilidade de criação destes hospedeiros, que acabam por reduzir os custos de produção (Parra 1986; 2002).

No entanto, existem divergências na escolha do hospedeiro mais adequado. Lewis et al. (1976) demonstraram que $S$. cerealella é nutricionalmente menos adequada para multiplicação de Trichogramma em relação às outras espécies, recomendando sua substituição por A. kuehniella. Resultados semelhantes têm sido encontrados no Brasil. Nestes estudos, fica evidente que o melhor hospedeiro pode ser variável, dependendo da espécie (Parra \& Zucchi 2004), podendo ser A. kuehniella para umas e C. cephalonica para as outras espécies. 
Trichogramma atopovirilia Oatman \& Platner, 1983, Trichogrammatoidea annulata De Santis,1972 e Trichogramma bruni Nagaraja, 1983 são espécies neotropicais com potencial para uso em programas de controle biológico no Brasil. T. atopovirilia, para o controle de Gymnandrosoma aurantianum Lima, 1927 (Lepidoptera, Torticidae) em citros (Molina et al. 2005); Diaphania hyalinata L. (Melo et al. 2007) (Lepidoptera, Crambidae), Anticarsia gemmatalis Hübner, 1818 (Lepidoptera, Noctuidae) (Cañete \& Foerster 2003) e Spodoptera frugiperda (Lepidoptera, Noctuidae) (J.E. Smith) (Beserra \& Parra 2004).

Além disso, T. atopovirilia e T. annulata apresentam potencial de controle para Stenoma catenifer Walsingham, 1912 (Lepidoptera, Elachistidae), em abacateiro, em condições de semi-campo (Nava et al. 2007). As espécies T. bruni e T. annulata foram registradas sobre ovos de $S$. catenifer e Hypocala andremona (Lepidoptera, Noctuidae), em níveis de parasitismo natural de até 40 e $50 \%$ dos ovos da praga, respectivamente (Hohmann \& Lovato 2003). Para que estas espécies possam ser utilizadas em programas de Controle Biológico, faz-se necessária a seleção do hospedeiro alternativo mais adequado para a sua criação massal. Sendo assim, o objetivo deste trabalho foi selecionar, entre três hospedeiros alternativos, aquele que permita o melhor desenvolvimento das espécies T. atopovirilia; T. bruni e $T$. annulata, utilizando-se como parâmetro comparativo as tabelas de vida de fertilidade nos respectivos hospedeiros.

\section{MATERIAL E MÉTODOS}

Criação dos parasitóides. Os insetos utilizados nos experimentos foram criados no Laboratório de Biologia dos Insetos do Departamento de Entomologia e Acarologia, da Escola Superior de Agricultura Luiz de Queiroz (Esalq), em Piracicaba, São Paulo.

T. bruni foi coletada em ovos de Heliconius erato phyllis (Fabricius), em Piracicaba, São Paulo; T. atopovirilia, obtida no Laboratório de Entomologia da Embrapa Semi-Árido, Petrolina, Pernambuco; e T. annulata coletada em ovos de $S$. catenifer, em Londrina, Paraná.

Para evitar possível condicionamento pré-imaginal ao hospedeiro de criação, antes da instalação dos experimentos, as espécies que até então vinham sendo mantidas em ovos de A. kuehniella, foram criadas por três gerações sucessivas em ovos dos seus respectivos hospedeiros naturais G. aurantianum para T. atopovirilia e S. catenifer para T. bruni e para $T$. annulata. A partir dos adultos da geração F3 provenientes dos hospedeiros naturais, cada espécie de parasitóide foi criada em três espécies de hospedeiros alternativos: 1) $C$. cephalonica; 2) A. kuehniella; e 3) S. cerealella.

As populações dos parasitóides estudados foram mantidas em seus hospedeiros alternativos de acordo com Parra (1997), sendo A. kuehniella criada em dieta à base de farinha de trigo integral (97\%) e levedura de cerveja (3\%) (Parra 1997), C. cephalonica em dieta à base de germe de trigo $(97 \%) \mathrm{e}$ levedura de cerveja (3\%) (Bernardi et al. 2000) e S. cerealella em grãos de trigo (Haji et al. 2002).
Procedimentos experimentais. Fêmeas de 12-24 h de idade, provenientes dos ovos de cada hospedeiro alternativo, foram individualizadas em tubos de vidro $(12 \times 75 \mathrm{~mm})$, e alimentadas com uma gotícula de mel puro. Para cada fêmea foram oferecidos cartões com 60 ovos (com idade de 0-24 h) dos respectivos hospedeiros, sendo os cartões substituídos a cada $24 \mathrm{~h}$ até a morte da fêmea. Os experimentos foram realizados na $1^{\mathrm{a}}, 10^{\mathrm{a}}$ e $28^{\mathrm{a}}$ gerações, em condições controladas (B.O.D: $25 \pm 1{ }^{\circ} \mathrm{C}, 70 \pm 10 \%$ UR e fotofase de 14 horas). Foi estimada a duração do período ovo-adulto, viabilidade, razão sexual $(q / q+\widehat{T})$, capacidade de parasitismo (diária e total) e longevidade das fêmeas.

Com base nos resultados obtidos, foram construídas tabelas de vida de fertilidade, sendo $\mathrm{x}=$ ponto médio de cada idade das fêmeas parentais; $1 \mathrm{x}=$ expectativa de vida até a idade $\mathrm{x} ; \mathrm{mx}=$ fertilidade específica ou número de descendentes por fêmea produzidos na idade $\mathrm{x}$ e que originarão fêmeas; $1 \mathrm{xmx}$ = número total de fêmeas nascidas na idade $\mathrm{x}$. Baseandose nas informações da tabela de vida, foram estimados os seguintes parâmetros para cada tratamento: $\mathrm{R}_{\mathrm{o}}$ (taxa líquida de reprodução, ou seja, a taxa de aumento populacional a cada geração), MGT (tempo médio de geração), $\mathrm{r}_{\mathrm{m}}$ (taxa intrínseca de crescimento), $\lambda$ (taxa finita de aumento) e DT (tempo necessário para a população duplicar). Em seguida, estes valores foram utilizados para obtenção da taxa extrínseca de crescimento $\mathrm{r}_{\mathrm{m}}$ e do intervalo de gerações $\mathrm{T}$ pelo método interativo (Southwood 1995).

Os parâmetros da tabela de vida de fertilidade e respectivos erros padrão foram estimados através da técnica de "jacknife" (Meyer et al. 1986) e as médias comparadas pelo teste " $\mathrm{t}$ " unilateral, $(\mathrm{P} \leq 0,05)$, utilizando o software "Lifetable.sas" (Maia et al. 2000) no ambiente "SAS System".

Odelineamento experimental foi inteiramente casualizado, com 25 repetições ( 1 fêmea $=1$ repetição) por tratamento (cada hospedeiro alternativo $=1$ tratamento).

\section{RESULTADOS E DISCUSSÃO}

Deumaformageral,paraastrêsespéciesdetricogramatídeos estudadas, o intervalo médio entre gerações $(T)$, ou seja, a duração média do período entre o nascimento dos indivíduos de uma geração e o da geração seguinte foi significativamente superior quando os parasitóides foram provenientes de $S$. cerealella (Tabelas I, II e III). Especificamente para $T$. annulata, o valor de $\mathrm{T}$ foi aproximadamente um dia maior em $S$. cerealella do que o constatado quando os hospedeiros $C$. cephalonica e A. kuehniella foram utilizados.

$\mathrm{O}$ mesmo pode-se dizer para a taxa líquida de reprodução (Ro), que revela o número de vezes que a população aumenta a cada geração. Esta taxa foi, de uma forma geral, superior quando se utilizou o hospedeiro $C$. cephalonica ou $A$. kuehniella (Tabelas I, II e III), indicando que em S. cerealella os parasitóides têm, ao longo de sua vida, menor capacidade de gerar descendentes. Valores de Ro semelhantes foram registrados para Trichogramma exiguum Pinto \& Platner, 1978 (Oliveira et al. 2007); Trichogramma pretiosum Riley, 1879 e Trichogramma acacioi Brun, Moraes \& Soares, (Pratissoli 
Tabela I. Intervalo entre gerações (T), taxa líquida de reprodução (Ro), taxa intrínseca de crescimento $\left(\mathrm{r}_{\mathrm{m}}\right)$ e taxa finita de aumento $(\lambda)$ de $T$. annulata em diferentes hospedeiros alternativos ao longo de gerações sucessivas de laboratório $\left(25 \pm 1^{\circ} \mathrm{C}, 70 \pm 10 \%\right.$ UR e fotofase $\left.14 \mathrm{~h}\right)$.

\begin{tabular}{|c|c|c|c|}
\hline \multirow{2}{*}{ Geração } & \multicolumn{3}{|c|}{ Intervalo entre gerações $(\mathrm{T})$} \\
\hline & A. kuehniella & C. cephalonica & S. cerealella \\
\hline $1^{\mathrm{a}}$ & $12,96 \pm 0,198 \mathrm{Aa}$ & $11,70 \pm 0,136 \mathrm{Ba}$ & $13,35 \pm 0,122 \mathrm{Aa}$ \\
\hline $10^{\mathrm{a}}$ & $11,25 \pm 0,116 \mathrm{Bc}$ & $11,60 \pm 0,092 \mathrm{Ba}$ & $12,73 \pm 0,139 \mathrm{Ab}$ \\
\hline \multirow[t]{3}{*}{$28^{\mathrm{a}}$} & $12,33 \pm 0,136 \mathrm{Bb}$ & $11,94 \pm 0,097 \mathrm{Ca}$ & $13,16 \pm 0,099 \mathrm{Aa}$ \\
\hline & \multicolumn{3}{|c|}{ Taxa líquida de reprodução (Ro) } \\
\hline & A. kuehniella & C. cephalonica & S. cerealella \\
\hline $1^{\mathrm{a}}$ & $64,40 \pm 6,914 \mathrm{Aa}$ & $77,00 \pm 5,708 \mathrm{Ab}$ & $27,29 \pm 1,950 \mathrm{Bb}$ \\
\hline $10^{\mathrm{a}}$ & $52,15 \pm 2,612 \mathrm{Ba}$ & $74,02 \pm 3,322 \mathrm{Ab}$ & $33,28 \pm 2,314 \mathrm{Ca}$ \\
\hline \multirow[t]{3}{*}{$28^{\mathrm{a}}$} & $54,11 \pm 4,805 \mathrm{Ba}$ & $111,75 \pm 8,021 \mathrm{Aa}$ & $32,21 \pm 1,956 \mathrm{Ca}$ \\
\hline & \multicolumn{3}{|c|}{ Razão infinitesimal (rm) } \\
\hline & A. kuehniella & C. cephalonica & S. cerealella \\
\hline $1^{\mathrm{a}}$ & $0,33 \pm 0,006 \mathrm{Ba}$ & $0,35 \pm 0,004 \mathrm{Ab}$ & $0,26 \pm 0,004 \mathrm{Ca}$ \\
\hline $10^{\mathrm{a}}$ & $0,34 \pm 0,003 \mathrm{Ba}$ & $0,36 \pm 0,003 \mathrm{Aab}$ & $0,25 \pm 0,005 \mathrm{Ca}$ \\
\hline \multirow[t]{3}{*}{$28^{\mathrm{a}}$} & $0,31 \pm 0,006 \mathrm{Bb}$ & $0,37 \pm 0,006 \mathrm{Aa}$ & $0,26 \pm 0,004 \mathrm{Ca}$ \\
\hline & \multicolumn{3}{|c|}{ Razão finita de aumento $(\lambda)$} \\
\hline & A. kuehniella & C. cephalonica & S. cerealella \\
\hline $1^{\mathrm{a}}$ & $1,39 \pm 0,008 \mathrm{Bb}$ & $1,43 \pm 0,006 \mathrm{Ab}$ & $1,30 \pm 0,005 \mathrm{Ca}$ \\
\hline $10^{\mathrm{a}}$ & $1,41 \pm 0,004 \mathrm{Ba}$ & $1,43 \pm 0,005 \mathrm{Ab}$ & $1,29 \pm 0,006 \mathrm{Ca}$ \\
\hline $28^{\mathrm{a}}$ & $1,36 \pm 0,008 \mathrm{Bb}$ & $1,46 \pm 0,009 \mathrm{Aa}$ & $1,30 \pm 0,006 \mathrm{Ca}$ \\
\hline
\end{tabular}

Médias seguidas da mesma letra não diferem significativamente entre si pelo teste "t" unilateral $(\mathrm{P} \leq 0,05)$. Letras maiúsculas: comparação entre os hospedeiros. Letras minúsculas: comparação das gerações dentro de cada hospedeiro.

et al. 2004a); Trichogramma bournieri Pintureau \& Babault e Trichogramma sp. nr. mwanzai Schulten \& Feijen (Haile et al. 2002), também em ovos de S. cerealella.

Analisando os valores da razão intrínseca de crescimento populacional $\left(\mathrm{r}_{\mathrm{m}}\right)$ de $T$. annulata, obtiveram-se valores superiores no hospedeiro C. cephalonica quando comparado aos demais hospedeiros; para T. atopovirilia, os valores de $\mathrm{r}_{\mathrm{m}}$ foram superiores nos hospedeiros A. kuehniella e $C$. cephalonica, enquanto que para T. bruni em C. cephalonica os valores foram superiores. Para todas as espécies, os menores valores foram obtidos quando estes parasitóides foram provenientes de S. cerealella (Tabelas I, II e III).

A taxa intrínseca de aumento $\left(\mathrm{r}_{\mathrm{m}}\right)$ é o principal dado que se obtém ao fazer-se uma tabela de vida de fertilidade (Pedigo \& Zeiss 1996) e, segundo Andrewartha \& Birch (1954), quanto maior o valor de $\mathrm{r}_{\mathrm{m}}$ mais bem sucedida será a espécie, em um determinado ambiente. Desta forma, para T. annulata e T. bruni, C. cephalonica foi o hospedeiro mais indicado para um incremento populacional e criação, enquanto que para $T$. atopovirilia A. kuehniella e C. cephalonica foram os melhores e igualmente favoráveis. Desta forma, é de se esperar que para produção massal dos referidos parasitóides, visando a programas de Controle Biológico Aplicado, estes sejam os hospedeiros mais adequados.

A razão finita de aumento $(\lambda)$, responsável pela indicação do número de fêmeas que são adicionadas à população por cada fêmea, apresentou diferenças significativas entre os hospedeiros. Para T. annulata e T. bruni, os maiores valores foram registrados em C. cephalonica, enquanto que para
T. atopovirilia os maiores valores foram registrados em $A$. kuehniella e C. cephalonica. Seguindo a tendência geral, em todos os casos os menores valores foram obtidos quando estes parasitóides foram provenientes de $S$. cerealella (Tabelas I, II e III).

Para todas as espécies foram observadas diferenças nos valores de Ro, $r_{m}$ e $\lambda$ após gerações sucessivas no mesmo hospedeiro. Para T. annulata, em C. cephalonica, hospedeiro em que observou-se maior crescimento populacional, os valores de Ro, $r_{m}$ e $\lambda$ tenderam a aumentar ao longo das gerações, evidenciando uma aparente adaptação desta espécie neste hospedeiro. Nos demais hospedeiros, estes valores permaneceram constantes ou diminuíram (Tabela I). Para T. atopovirilia e T. bruni os resultados foram semelhantes; no entanto, quando estes parasitóides foram provenientes de $S$. cerealella os valores de $\mathrm{Ro}, \mathrm{r}_{\mathrm{m}}$ e $\lambda$ tenderam a diminuir com o passar das gerações. Interessante é notar que para $T$. bruni estes valores também diminuíram em A. kuehniella, hospedeiro sugerido como sendo adequado para algumas espécies de tricogramatídeos (Parra \& Zucchi 2004).

Os resultados demonstraram que os parâmetros obtidos da tabela de vida de fertilidade podem variar de acordo com a espécie hospedeira utilizada, e que estas informações são úteis para indicar o hospedeiro alternativo mais adequado para criação de T. atopovirilia, T. bruni e T. annulata. Muitos trabalhos demonstram a influência de fatores abióticos sobre o potencial reprodutivo de parasitóides (Pratissoli et al. 2004a; 2004b, 2007); todavia, fatores bióticos, como o hospedeiro alternativo de criação, são normalmente negligenciados,

Tabela II. Intervalo entre gerações (T), taxa líquida de reprodução (Ro), taxa intrínseca de crescimento $\left(\mathrm{r}_{\mathrm{m}}\right)$ e taxa finita de aumento $(\lambda)$ de T. atopovirilia em diferentes hospedeiros alternativos ao longo de gerações sucessivas de laboratório $\left(25 \pm 1^{\circ} \mathrm{C}, 70 \pm 10 \%\right.$ UR e fotofase $\left.14 \mathrm{~h}\right)$.

\begin{tabular}{|c|c|c|c|}
\hline \multirow{2}{*}{ Geração } & \multicolumn{3}{|c|}{ Intervalo entre gerações $(\mathrm{T})$} \\
\hline & A. kuehniella & C. cephalonica & S. cerealella \\
\hline $1^{\mathrm{a}}$ & $11,79 \pm 0,148 \mathrm{Aa}$ & $11,86 \pm 0,166 \mathrm{Aa}$ & $12,08 \pm 0,092 \mathrm{Ab}$ \\
\hline $10^{\mathrm{a}}$ & $11,32 \pm 0,117 \mathrm{Ba}$ & $11,37 \pm 0,141 \mathrm{Ba}$ & $13,48 \pm 0,169 \mathrm{Aa}$ \\
\hline \multirow[t]{3}{*}{$28^{\mathrm{a}}$} & $11,10 \pm 0,126 \mathrm{Ba}$ & $10,94 \pm 0,167 \mathrm{Bb}$ & $13,39 \pm 0,138 \mathrm{Aa}$ \\
\hline & \multicolumn{3}{|c|}{ Taxa líquida de reprodução (Ro) } \\
\hline & A. kuehniella & C. cephalonica & S. cerealella \\
\hline $1^{\mathrm{a}}$ & $77,74 \pm 6,904 \mathrm{Ab}$ & $96,74 \pm 8,778 \mathrm{Ab}$ & $28,68 \pm 3,110 \mathrm{Ba}$ \\
\hline $10^{\mathrm{a}}$ & $79,27 \pm 6,642 \mathrm{Bb}$ & $130,43 \pm 6,672 \mathrm{Aa}$ & $24,52 \pm 2,074 \mathrm{Ca}$ \\
\hline \multirow[t]{3}{*}{$28^{\mathrm{a}}$} & $106,96 \pm 6,655 \mathrm{Aa}$ & $115,78 \pm 8,921 \mathrm{Aa}$ & $16,87 \pm 1,706 \mathrm{Bb}$ \\
\hline & \multicolumn{3}{|c|}{ Razão infinitesimal (rm) } \\
\hline & A. kuehniella & C. cephalonica & S. cerealella \\
\hline $1^{\mathrm{a}}$ & $0,37 \pm 0,007 \mathrm{Aa}$ & $0,37 \pm 0,006 \mathrm{Aa}$ & $0,30 \pm 0,010 \mathrm{Ba}$ \\
\hline $10^{\mathrm{a}}$ & $0,37 \pm 0,005 \mathrm{Aa}$ & $0,36 \pm 0,002 \mathrm{Bb}$ & $0,28 \pm 0,006 \mathrm{Cb}$ \\
\hline \multirow[t]{3}{*}{$28^{\mathrm{a}}$} & $0,36 \pm 0,006 \mathrm{Ba}$ & $0,38 \pm 0,003 \mathrm{Aa}$ & $0,25 \pm 0,008 \mathrm{Cc}$ \\
\hline & \multicolumn{3}{|c|}{ Razão finita de aumento $(\lambda)$} \\
\hline & A. kuehniella & C. cephalonica & S. cerealella \\
\hline $1^{\mathrm{a}}$ & $1,43 \pm 0,010 \mathrm{Aa}$ & $1,46 \pm 0,009 \mathrm{Aa}$ & $1,35 \pm 0,013 \mathrm{Ba}$ \\
\hline $10^{\mathrm{a}}$ & $1,45 \pm 0,008 \mathrm{Aa}$ & $1,43 \pm 0,003 \mathrm{Bb}$ & $1,32 \pm 0,008 \mathrm{Cb}$ \\
\hline $28^{\mathrm{a}}$ & $1,44 \pm 0,009 \mathrm{Aa}$ & $1,47 \pm 0,005 \mathrm{Ba}$ & $1,28 \pm 0,010 \mathrm{Cc}$ \\
\hline
\end{tabular}

Médias seguidas da mesma letra não diferem significativamente entre si pelo teste " $t$ " unilateral $(\mathrm{P} \leq 0,05)$. Letras maiúsculas: comparação entre os hospedeiros. Letras minúsculas: comparação das gerações dentro de cada hospedeiro. 
Tabela III. Intervalo entre gerações (T), taxa líquida de reprodução (Ro), taxa intrínseca de crescimento $\left(\mathrm{r}_{\mathrm{m}}\right)$ e taxa finita de aumento $(\lambda)$ de $T$. bruni em diferentes hospedeiros alternativos ao longo de gerações sucessivas de laboratório $\left(25 \pm 1^{\circ} \mathrm{C}, 70 \pm 10 \%\right.$ UR e fotofase $\left.14 \mathrm{~h}\right)$.

\begin{tabular}{|c|c|c|c|}
\hline \multirow{2}{*}{ Geração } & \multicolumn{3}{|c|}{ Intervalo entre gerações $(\mathrm{T})$} \\
\hline & A. kuehniella & C. cephalonica & S. cerealella \\
\hline $1^{\mathrm{a}}$ & $11,85 \pm 0,077 \mathrm{Bb}$ & $11,83 \pm 0,209 \mathrm{Ba}$ & $12,37 \pm 0,189 \mathrm{Ab}$ \\
\hline $10^{\mathrm{a}}$ & $11,84 \pm 0,330 \mathrm{Bb}$ & $11,98 \pm 0,122 \mathrm{Ba}$ & $13,20 \pm 0,317 \mathrm{Aa}$ \\
\hline \multirow[t]{3}{*}{$28^{\mathrm{a}}$} & $12,26 \pm 0,199 \mathrm{Ba}$ & $11,80 \pm 0,132 \mathrm{Ba}$ & $13,11 \pm 0,224 \mathrm{Aa}$ \\
\hline & \multicolumn{3}{|c|}{ Taxa líquida de reprodução (Ro) } \\
\hline & A. kuehniella & C. cephalonica & S. cerealella \\
\hline $1^{\mathrm{a}}$ & $47,07 \pm 3,954 \mathrm{Aa}$ & $27,58 \pm 3,886 \mathrm{Bb}$ & $14,72 \pm 1,949 \mathrm{Ca}$ \\
\hline $10^{\mathrm{a}}$ & $28,55 \pm 5,339 \mathrm{Bb}$ & $46,93 \pm 4,218 \mathrm{Aa}$ & $13,72 \pm 2,344 \mathrm{Ca}$ \\
\hline \multirow[t]{3}{*}{$28^{\mathrm{a}}$} & $23,25 \pm 2,925 \mathrm{Bb}$ & $40,02 \pm 2,924 \mathrm{Aa}$ & $6,71 \pm 0,903 \mathrm{Cb}$ \\
\hline & \multicolumn{3}{|c|}{ Razão infinitesimal (rm) } \\
\hline & A. kuehniella & C. cephalonica & S. cerealella \\
\hline $1^{\mathrm{a}}$ & $0,31 \pm 0,006 \mathrm{Aa}$ & $0,28 \pm 0,008 \mathrm{Bb}$ & $0,22 \pm 0,010 \mathrm{Ca}$ \\
\hline $10^{\mathrm{a}}$ & $0,28 \pm 0,012 \mathrm{Bb}$ & $0,32 \pm 0,007 \mathrm{Aa}$ & $0,19 \pm 0,011 \mathrm{Cb}$ \\
\hline \multirow[t]{3}{*}{$28^{\mathrm{a}}$} & $0,25 \pm 0,010 \mathrm{Bb}$ & $0,31 \pm 0,005 \mathrm{Aa}$ & $0,14 \pm 0,010 \mathrm{Cc}$ \\
\hline & \multicolumn{3}{|c|}{ Razão finita de aumento $(\lambda)$} \\
\hline & A. kuehniella & C. cephalonica & S. cerealella \\
\hline $1^{\mathrm{a}}$ & $1,36 \pm 0,008 \mathrm{Aa}$ & $1,32 \pm 0,011 \mathrm{Bb}$ & $1,25 \pm 0,013 \mathrm{Ca}$ \\
\hline $10^{\mathrm{a}}$ & $1,32 \pm 0,016 \mathrm{Bb}$ & $1,37 \pm 0,009 \mathrm{Aa}$ & $1,21 \pm 0,014 \mathrm{Ca}$ \\
\hline $28^{\mathrm{a}}$ & $1,29 \pm 0,013 \mathrm{Bc}$ & $1,36 \pm 0,007 \mathrm{Aa}$ & $1,15 \pm 0,012 \mathrm{Cb}$ \\
\hline
\end{tabular}

Médias seguidas da mesma letra não diferem significativamente entre si pelo teste " $\mathrm{t}$ " unilateral $(\mathrm{P} \leq 0,05)$. Letras maiúsculas: comparação entre os hospedeiros. Letras minúsculas: comparação das gerações dentro de cada hospedeiro.

a despeito de também afetarem este potencial, como foi demonstrado neste trabalho.

Em laboratórios de criação massal, é interessante que se obtenha o maior número de insetos no menor tempo possível. No caso de parasitóides, conhecer o hospedeiro que proporciona a maior capacidade de crescimento populacional é imprescindível à obtenção de um sistema de criação com características desejáveis.

Estudos com estes parasitóides, em hospedeiros alternativos, têm demonstrado o seu alto potencial reprodutivo, algumas vezes superior quando criados nos seus hospedeiros naturais (Dias et al. 2008). Para T. annulata, estes autores observaram um maior número de ovos parasitados de $C$. cephalonica $(53,36)$, durante 24 horas, ao relatado por Nava et al. (2007) no hospedeiro natural $S$. catenifer $(41,26)$, nas mesmas condições ambientais. Já $T$. atopovirilia, parasitou uma média de 186,80 ovos em C. cephalonica e 143,92 ovos em A. kuehniella, enquanto em G. aurantianum hospedeiro natural obtiveram-se 105,80 ovos (Molina et al., 2005). Para T. bruni observou-se uma média de 78,60 e 60,21 ovos em $C$. cephalonica e $A$. kuehniella, respectivamente, e 45,48 ovos em $S$. catenifer.

Assim, os resultados demonstram a viabilidade destes hospedeiros alternativos, para a criação massal destes parasitóides. Além disso, a utilização de Trichogramma spp. tem sido feita com a escolha de um hospedeiro alternativo, pela facilidade de criação ou disponibilidade. Entretanto, é necessário se determinar o hospedeiro alternativo mais adequado para cada espécie, pois muitas vezes o insucesso de um programa de controle biológico pode estar relacionado à escolha inadequada do hospedeiro de criação massal.

\section{CONCLUSÕES}

1. Corcyra cephalonica é o hospedeiro alternativo mais adequado para Trichogrammatoidea annulata e Trichogramma bruni, enquanto Trichogramma atopovirilia pode ser criado em Anagasta kuehniella ou Corcyra cephalonica, ao longo das gerações.

2. Sitotroga cerealella é o hospedeiro menos adequado para a criação das espécies de tricogramatídeos estudadas.

\section{REFERÊNCIAS}

Andrewartha, H. G. \& L. C. Birch. 1954. The innate capacity for increase in numbers, p. 31-54. In: H. G. Andrewartha \& L. C. Birch (eds.). The distribution and abundance of animals. Chicago, University of Chicago Press, $793 \mathrm{p}$.

Bai, B.; R. F. Luck; L. Forster; B. Stephens \& J. A. M. Janssen. 1992. The effect of host size on quality attributes of the egg parasitoid, Trichogramma pretiosum. Entomologia Experimentalis et Applicata 64: $37-48$

Bernardi, E. B.; M. L. Haddad \& J. R. P. Parra. 2000. Comparison of artificial diets for rearing Corcyra cephalonica (Stainton, 1865) (Lep., Pyralidae) for Trichogramma mass production. Revista Brasileira de Biologia 60: 45-52.

Beserra, E. B. \& J. R. P. Parra. 2004. Biologia e parasitismo de Trichogramma atopovirilia Oatman \& Platner e Trichogramma pretiosum Riley (Hymenoptera, Trichogrammatidae) em ovos de Spodoptera frugiperda (J. E. Smith) (Lepidoptera, Noctuidae). Revista Brasileira de Entomologia 48: 119-126.

Cañete, C. L \& L. A. Foerster. 2003. Incidência natural e biologia de Trichogramma atopovirilia Oatman \& Platner, 1983 (Hymenoptera, Trichogrammatidae) em ovos de Anticarsia gemmatalis Hübner, 1818 (Lepidoptera, Noctuidae). Revista Brasileira de Entomologia 47: 201-204.

Dias, N. S.; J. R. P. Parra \& T. C. C. Lima. 2008. Seleção de hospedeiro alternativo para três espécies de tricogramatídeos neotropicais. Pesquisa Agropecuária Brasileira 43: 1467-1473.

Haji, F. N. P.; L. Prezotti; J. S. Carneiro \& J. A. Alencar. 2002. Trichogramma pretiosum para o controle de pragas no tomateiro industrial, p. 477-491. In: J. R. P. Parra; P. S. M. Botelho; B. S. Corrêa-Ferreir \& J. M. S. Bento (eds.). Controle biológico no Brasil, parasitóides e predadores. Barueri, Manole, $635 \mathrm{p}$.

Haile, A. T.; S. A. Hassan; S. Sithanantham; C. K. P. O. Ogol \& J. Baungärtner. 2002. Comparative life table analysis of Trichogramma bournieri Pintureau and Babault and Trichogramma sp. nr. mwanzai Schulten and Feijen (Hym., Trichogrammatidae) from Kenya. Journal of Applied Entomology 127: 287-292.

Hoffmann, M.; P. R. Ode; D. L. Walker; J. Gardner; S. Van nouhuys \& A. M. Shelton. 2001. Performance of Trichogramma ostriniae (Hymenoptera: Trichogrammatidae) reared on factitious hosts, including the target host, Ostrinia nubilalis (Lepidoptera: Crambidae). Biological Control 21: $1-10$.

Hohmann, C. L \& L. Lovato. 2003. Parasitism of Hypocala andremona (Stoll) (Lepidoptera: Noctuidae) eggs on persimmon trees by Trichogrammatids. Neotropical Entomology 32: 351-353.

Lewis, W. J.; H. R. Gross Jr.; W. D. Perkins; E. F. Knipling \& J. Voegelé. 1976. Production and performance of Trichogramma reared on eggs of Heliothis zea and other hosts. Environmental Entomology 5: 449-452.

Maia, H. N. M.; A. J. B. Luiz \& C. Campanhola. 2000. Statistical inference on associated fertility life table parameters using jackknife technique: computational aspects. Journal of Economic Entomology 93: 511-518

Meyer, J. S.; C. G. Ingersoll; L. L. Mcdonald \& M. S. Boyce. 1986. Estimating uncertainty in population growth rates: jackknife vs. 
Bootstrap techniques. Ecology 67: 1156-1166.

Melo, R. L.; D. Pratissoli; R. A. Polanczyk; D. F. Melo; R. Barros \& A. M. Milanez. 2007. Biologia e Exigências Térmicas de Trichogramma atopovirilia Oatman \& Platner (Hymenoptera: Trichogrammatidae) em ovos de Diaphania hyalinata L. (Lepidoptera: Pyralidae). Neotropical Entomology 36: 431-435.

Molina, R. M. S.; V. Fronza \& J. R. P. Parra. 2005. Seleção de Trichogramma spp., para o controle de Ecdytolopha aurantiana com base na biologia e exigências térmicas. Revista Brasileira de Entomologia 49: 151-158.

Nava, D. E.; K. M. Takahashi \& J. R. P. Parra. 2007. Linhagens de Trichogramma e Trichogrammatoidea para controle de Stenoma catenifer. Pesquisa Agropecuária Brasileira 42: 9-16.

Oliveira, H. N.; D. Pratissoli; C. A. Colombi; R. A. Polanczyk \& L. P. Dalvi. 2007. Tabela de vida de fertilidade de Trichogramma exiguum (Hymenoptera: Trichogrammatidae). Idesia 25: 73-76.

Parra, J. R. P. 1986. Criação de insetos para estudos com patógenos, p. 348373. In: S. B. Alves (eds.). Controle Microbiano de Insetos. São Paulo, Manole, $407 \mathrm{p}$.

Parra, J. R. P. 1997. Técnicas de criação de Anagasta kuehniella, hospedeiro alternativo para produção de Trichogramma, p. 121-150. In: J. R. P. Parra \& R.A. Zucchi (eds.). Trichogramma e o controle biológico aplicado. Piracicaba, FEALQ, $324 \mathrm{p}$.

Parra, J. R. P. 2002. Criação massal de inimigos naturais, p. 143-161. In: J. R. P. Parra; P. S. M. Botelho; B. S. Corrêa-Ferreira \& J. M. S. Bento (eds.). Controle biológico no Brasil, parasitóides e predadores. Barueri, Manole, $609 \mathrm{p}$.

Parra, J. R. P. \& R. A. Zucchi. 2004. Trichogramma in Brazil: Feasibility of use after twenty years of research. Neotropical Entomology 33: 271-281.
Pedigo, L. P. \& M. R. Zeiss. 1996. Developing a degree-day model for predicting insect development, p. 67-74. In: L. P. Pedigo \& M. R. Zeiss (eds.). Analyses in insect ecology and management. Ame,: Iowa State University Press, $168 \mathrm{p}$.

Pratissoli, D.; O. A. Fernandes; J. C. Zanuncio \& P. L. Pastori. 2004a. Fertility life table of Trichogramma pretiosum and Trichogramma acacioi (Hymenoptera: Trichogrammatidae) on Sitotroga cerealella (Lepidoptera: Gelechiidae) eggs at different constant temperatures. Annals of the Entomological Society of America 94: 729-731

Pratissoli, D.; J. Zanuncio; U. R. Vianna; J. S. Andrade; E. M. Guimarães \& M. C. Espindula. 2004b. Fertility life table of Trichogramma pretiosum and Trichogramma acacioi on eggs of Anagasta kuehniella at different temperatures. Pesquisa Agropecuária Brasileira 39: 193-196.

Pratissoli, D.; R. A. Polanczyk; G. S. Andrade; A. M. Holtz; A. F. Silva \& P. L. Pastori. 2007. Tabela de vida de fertilidade de cinco linhagens de Trichogramma pretiosum Riley (Hym.: Trichogrammatidae) criadas em ovos de Tuta absoluta (Merick) (Lep.: Gelechiidae), sob temperaturas constantes e alternadas. Ciência Rural 37: 618-622.

Roriz, V.; L. Oliveira \& P. Garcia. 2006. Host suitability and preference studies of Trichogramma cordubensis (Hymenoptera: Trichogrammatidae). Biological Control 36: 331-336.

Rukmowati-Brotodjojo, R. R. \& R. R. Walter. 2006. Oviposition and reproductive performance of a generalist parasitoid (Trichogramma pretiosum) exposed to host species that differ in their physical characteristics. Biological Control 39: 300-312.

Schmidt, J. M. 1994. Host recognition and acceptance by Trichogramma, p. 165-199. In: E. Wajnberg \& S. A. Hassan (eds.). Biological control with egg parasitoids. Wallingford, $C A B$ International, $304 \mathrm{p}$.

Southwood, T. R. E. 1995. Ecological methods. London, Chapmam \& Hall, $524 \mathrm{p}$. 